\title{
Molecular detection and antibiotic resistance pattern of extended-spectrum beta-lactamase producing Escherichia coli in a Tertiary Hospital in Enugu, Nigeria
}

Ifeyinwa N. Nwafia*, Martin E. Ohanu, Samuel O. Ebede and Uchenna C. Ozumba

\begin{abstract}
Background: The use of antibiotic agents in the treatment of infectious diseases has greatly contributed to the decrease in morbidity and mortality, but these great advances in treatment are being undermined by the rapidly increasing antimicrobial resistant organisms. Extended-spectrum beta-lactamases are enzymes hydrolyzing the beta lactam antibiotics, including third generation cephalosporins and monobactams but not cephamycins and carbapenems. They pose a serious global health threat and have become a challenge for health care providers. The aim of this research was to assess the prevalence of extended-spectrum beta-lactamase producing Escherichia coli in University of Nigeria Teaching Hospital Ituku-Ozalla Enugu and to detect the risk factors for acquisition of the resistant organism. To proffer advice on antibiotic stewardship in clinical practice and public health interventions, to curb the spread of the resistant organisms in the hospital.

Results: Out of the 200 E. coli isolates, 70 (35.00\%) were confirmed positive for extended-spectrum beta-lactamase production. Fifty-three (75.7\%) were from hospital acquired infections. All the isolates were resistant to ampicillin, tetracycline and chloramphenicol while $68(97.14 \%)$ of the 70 isolates were susceptible to imipenem. Bla $a_{\mathrm{TEM}}, b / a_{\mathrm{SHV}}$ and bla $a_{\text {TEM }}$ were detected in $66(94 \%)$ of the 70 isolates. The ESBL bla genes detected were bla bla $a_{\text {TEM }}(n=7 ; 10.00 \%), b l a_{\text {SHV }}(n=2 ; 2.86 \%)$, bla $a_{\text {CTX-M } / T E M}(n=7 ; 10.0 \%)$, bla $a_{\text {CTX-M } / \mathrm{HHV}}(n=14 ; 20.0 \%)$ and bla $a_{\text {CTX-M } / T E M / S H V}$ $(n=10 ; 14.29 \%)$. The three bla genes were not detected in $4(5.71 \%)$ of the isolates. Recent surgery, previous antibiotic and intensive care unit admission were the associated risk factors to infections caused by extended-spectrum betalactamase producing E. coli.
\end{abstract}

Conclusion: There is a high rate of infections caused by extended-spectrum beta-lactamase producing E. coli. Recent surgery, previous antibiotic and intensive care unit admission were associated risk factors.

Keywords: Antimicrobial resistance, Escherichia coli, Extended-spectrum beta-lactamase, Molecular detection, Risk factors

\section{Background}

Escherichia coli (E. coli) is a common enteric organism and one of the predominant species in most bacterial

\footnotetext{
*Correspondence: ifeyinwa.nwafia@unn.edu.ng
}

Department of Medical Microbiology, Faculty of Medical Sciences,

College of Medicine, University of Nigeria, Ituku-Ozalla Campus, Enugu

State, Nigeria infections [1]. Extended-spectrum beta-lactamase (ESBL) producing E. coli strains are causing global public health threats. They are resistant to several classes of antibiotics [2], which results in limited therapeutic options to treat the infections caused by these pathogens. The rapid increase in the spread of these antimicrobial resistant organisms, coupled with the unavailability of effective antimicrobial agents has made the World Health 
Organization (WHO) to warn against "post-antimicrobial era", where people die because of common infections and minor injuries [3]. Extended-spectrum beta-lactamases (ESBLs) are plasmid-encoded enzymes and are easily transferred from one bacterium to another by horizontal gene transfer. Horizontal gene transfer by these plasmid exchanges between $E$. coli strains is a recognized source of rapid spread of antimicrobial resistant strains [4]. The most frequently detected and clinically important ESBLs belong to the Temoniera (TEM), sulfhydryl variable (SHV) and cefotaximase-Munich (CTX-M) families [5]. The burden of infections caused by these resistant strains is enormous. These strains are associated with a higher mortality rate, increased length of hospital stay and increased health costs $[6,7]$. Owing to these consequences, coupled with the increasing incidence, it is important to carry out continuous surveillance on ESBL producing E. coli in the hospital. Therefore, this study was undertaken to investigate antibiotic resistance patterns, risk factors and molecular detection of $b l a_{\mathrm{CTX}-\mathrm{M}}$, $b l a_{\mathrm{TEM}}$ and $b l a_{\mathrm{SHV}}$ genes in $E$. coli in University of Nigeria Teaching Hospital Ituku-Ozalla, Enugu, with a view to providing comprehensive and reliable epidemiological information which will be used in the improvement of patient care and advice on antibiotic stewardship in clinical practice.

\section{Methods}

\section{Study design and setting}

This cross sectional study was conducted in Department of Medical Microbiology at the University of Nigeria Teaching Hospital (UNTH) Ituku-Ozalla, Enugu from January 2016 to May 2017. UNTH Ituku-Ozalla, Enugu is situated in Enugu state which is one of the five states in the south-east geopolitical zone of Nigeria. The hospital is a 450 bed tertiary healthcare institution with facilities for comprehensive pediatrics and adult healthcare services, including intensive care unit.

\section{Patient}

The study involved 200 admitted patients in UNTH, Enugu. All consenting admitted adult ( $\geq 18$ years) patients whose culture results were positive for $E$. coli within the study period were included in the survey. To calculate the number of samples required to estimate the prevalence of ESBL producing E. coli in the hospital, we used one-sample Z-test with an estimated prevalence of $11 \%$, a confidence interval of $95 \%$ and maximum tolerable error of $10 \%$. This gave a minimum sample size of 120 samples. The clinical samples analyzed were urine (106), pleural and peritoneal aspirate (24), blood (4), wound swabs (53) and cerebro-spinal fluids (13).

\section{Patient involvement}

Patients were not directly involved, but their data were collected with a pretested structured questionnaire on their clinical records, after obtaining a written informed consent from them or their relatives. The questionnaire comprised four sections namely, demographics, history of present illness, past medical and co-morbidity history, devices utilization and past antibiotic used. The following data were then collected; ward admitted, age, gender, underlying disease, site of infection (from which E. coli was isolated), recent surgical procedure (within 3 months), immunosuppressant use within 1 month, parenteral nutrition and the presence of an indwelling urinary catheter and/or percutaneous tubes. Also previous antibiotic use (past 3 months) and the antibiotic taken were assessed. The investigator informed the patients and their relatives about their results and specific key infection prevention and control measures on how to prevent the spread of the resistant genes.

\section{Bacterial isolation and antibiotic susceptibility testing}

All the samples collected were cultured on MacConkey and 5\% sheep Blood agar plates (Oxoid Laboratories, Cambridge UK) and incubated aerobically at $37{ }^{\circ} \mathrm{C}$ for $24 \mathrm{~h}$. All suspected isolates with growth characteristics of $E$. coli were subjected to standard bacteriological identification methods and confirmed with API 20E confirmation system (Biomerieux, Marcy-Etoile, France).

Antibiotic susceptibility testing with 17 antibiotic agents (ceftazidime, cefotaxime, amoxicillin plus clavulanic acid, aztreonam, gentamicin, amikacin, ciprofloxacin, ofloxacin, nitrofurantoin, piperacillin/tazobactam, chloramphenicol, ampicillin, tetracycline, ampicillin, ertapenem, imipenem and meropenem (Oxoid, Cambridge, UK) were performed on Muller Hinton agar plates by disc diffusion method. A maximum of five antibiotic discs were placed on each plate for each isolated $E$. coli strain and then incubated at $37{ }^{\circ} \mathrm{C}$ for $24 \mathrm{~h}$, and the results recorded by measuring the inhibition zone diameter across the disc with a caliper and interpreted according to the Clinical and Laboratory Standard Institute guideline [8]. The quality control strains used were $E$. coli American type culture collection (ATCC) 25922 (ESBL negative strain) and E. coli ATCC 700603 (ESBL positive strain). E. coli isolates that were not susceptible to any of the third-generation cephalosporins were identified as potential ESBL producers and were then confirmed with chromogenic agar method. (Oxoid, Cambridge, UK). Seventy extended spectrum beta-lactamase producing $E$. coli confirmed isolates were immediately sub-cultured and 
preserved in a nutrient agar slant at $2-8{ }^{\circ} \mathrm{C}$ in a refrigerator and trypticase soy broth with $30 \%$ glycerol at $-70{ }^{\circ} \mathrm{C}$ in the freezer for subsequent molecular characterization.

\section{Molecular detection of the ESBL genes}

Molecular detection of $b l a_{\mathrm{CTX}-\mathrm{M}}, b l a_{\mathrm{TEM}}$ and $b l a_{\mathrm{SHV}}$ were done by multiplex polymerase chain reaction (PCR). The extraction of the DNA was done with mini-prep kit (Jena Bioscience, Jena, Germany) according to manufacturer's instructions. The primer used and their amplicon sizes were as shown in Table 1 . The PCR amplification was performed in $20 \mu \mathrm{l}$ reaction mixture containing the Solis Biodyne hot start Master mix (ready-to-load) containing $200 \mu \mathrm{M}$ each deoxynucleoside triphosphates (dNTP), $2 \mathrm{mM} \mathrm{MgCl}_{2}, 1 \times$ PCR Buffer, 2.0 units of TaqDNA polymerase, proof reading enzyme, $3 \mu \mathrm{l}$ of DNA (10-200 ng), and sterile nuclease-free water was used to make up the volume of the reaction mixture. The thermal cycling was conducted in an Eppendorf thermal cycler (Nexus series) at an initial denaturation of $95{ }^{\circ} \mathrm{C}$ for $15 \mathrm{~min}$, followed by 35 amplification cycles of $30 \mathrm{~s}$ at $95{ }^{\circ} \mathrm{C} ; 30 \mathrm{~s}$ at $60{ }^{\circ} \mathrm{C}$ and $1 \mathrm{~min}$ at $72^{\circ} \mathrm{C}$. This was followed by a final extension step of $72{ }^{\circ} \mathrm{C}$ for $10 \mathrm{~min}$. After amplification the product was separated on a $1.5 \%$ agarose gel electrophoresis and visualized by ethidium bromide staining. 100 base pair DNA ladders (Thermo Scientific) were used as DNA molecular weight standards.

Table 1 Primer sequences and their amplicon sizes

\begin{tabular}{lll}
\hline Primers & Nucleotide sequence & Base pair (bp) \\
\hline TEM-F & GCGGAACCCCTATTTG & 964 \\
TEM-R & TCTAAAGTATATATGAGTAAACTTGGTCTGAC & 964 \\
SHV-F & TTCGCCTGTGTATTATCTCCCTG & 854 \\
SHV-R & TTAGCGTTGCCAGTGYTCG & 854 \\
CTX-MF & ATGTGCAGYACCAGTAARGTKATGGC & 593 \\
CTX-M R & TGGGTRAARTARGTSACCAGAAYCAGCGG & 593
\end{tabular}

$n$ Number

\section{Statistical analysis}

All statistical analyses were performed using Statistical Package for Social Sciences (SPSS) computer software version 22. Descriptive analyses using percentages and frequencies were used for presence of antibiotic resistance pattern of extended-spectrum beta-lactamases and specific ESBL resistant genes. Continuous variables were compared with Chi square and multivariate logistic regression analysis to identify associated risk factors to ESBL producing $E$. coli infections. $P<0.05$ was considered statistically significant.

\section{Results}

During the study period, a total of $200 \mathrm{E}$. coli isolates were analysed from samples collected from patients with hospital 117 (58.5\%) and community 83 (41.5\%) acquired infections. Samples from female patients were 105 (52.5\%) and males 95 (47.5\%), with their ages ranging from 18 to 95 years old. Urine samples 106 (53.0\%) had the highest frequency followed by wound swab 53 (26.5\%), pleural and peritoneal aspirate $24(12.0 \%)$, cerebrospinal fluid 13 (6.5\%) and blood specimen 4 (2.00\%). Seventy $(35.0 \%)$ isolates were positive for extended spectrum beta lactamase production, 53 (75.7\%) from hospital acquired infections and 17 (24.3\%) from community acquired infections (Table 2).

Results of the antimicrobial susceptibility testing are shown in Table 3. Low rates of sensitivity were found against third generation cephalosporins \{Cefotaxime 5 (7.1\%), ceftazidime $7(10.00 \%)$, ceftriaxone $10(14.2 \%)\}$ and aztreonam 3 (4.2\%). Among the quinolones, sensitivity to ciprofloxacin and ofloxacin were $15(21.4 \%)$ and $26(37.1 \%)$ respectively. In aminoglycosides, sensitivity to amikacin $32(45.7 \%)$ was slightly higher than gentamicin 21 (30.00\%). Slightly more than half 39 (55.71\%) of the isolates were sensitive to nitrofurantoin. Sensitivity was highest with imipenem, 68 (97.1\%), followed by

Table 2 Sample distribution of ESBL positive and ESBL negative Escherichia coli from hospital and community acquired infections

\begin{tabular}{|c|c|c|c|c|c|c|c|}
\hline \multirow[t]{2}{*}{ Sample } & \multicolumn{3}{|l|}{ HAI } & \multicolumn{3}{|l|}{ CAI } & \multirow[t]{2}{*}{ Total } \\
\hline & ESBL (+) & ESBL (-) & Subtotal & ESBL (+) & ESBL (-) & Subtotal & \\
\hline Urine & 37 & 27 & 64 & 8 & 34 & 42 & $106(53.0)$ \\
\hline Urine & 5 & 28 & 33 & 5 & 15 & 20 & $53(26.5)$ \\
\hline $\begin{array}{l}\text { Pleural/Peritoneal } \\
\text { fluid }\end{array}$ & 3 & 8 & 11 & 3 & 10 & 13 & $24(12.0)$ \\
\hline CSF & 4 & 1 & 5 & 1 & 7 & 8 & $13(6.5)$ \\
\hline Blood & 4 & 0 & 4 & 0 & 0 & 0 & $4(2.0)$ \\
\hline Total & 53 & 64 & 117 & 17 & 66 & 83 & $200(100)$ \\
\hline
\end{tabular}

HAl hospital acquired infection, CAl community acquired infection, + positive, - negative, CSF cerebrospinal fluid 
Table 3 Antibiotic susceptibility pattern of ESBL producing Escherichia coli

\begin{tabular}{lccc}
\hline Antibiotics & S (\%) & I (\%) & R (\%) \\
\hline Amikacin & $32(45.71)$ & $6(8.71)$ & $32(45.43)$ \\
Ampicillin & $0(0.00)$ & $0(0.00)$ & $70(100.00)$ \\
Amoxicillin-clavulanic acid & $25(35.71)$ & $7(10.00)$ & $38(54.29)$ \\
Aztreonam & $3(4.29)$ & $0(0.00)$ & $67(95.71)$ \\
Ceftriaxone & $10(14.29)$ & $2(2.86)$ & $58(82.86)$ \\
Cefotaxime & $5(7.14)$ & $2(2.86)$ & $63(90.00)$ \\
Ceftazidime & $7(10.00)$ & $2(2.86)$ & $61(87.14)$ \\
Chloramphenicol & $0(0.00)$ & $0(0.00)$ & $70(100.00)$ \\
Ciprofloxacin & $15(21.43)$ & $3(4.29)$ & $52(74.29)$ \\
Ertapenem & $65(92.86)$ & $0(0.00)$ & $5(7.14)$ \\
Gentamicin & $21(30.00)$ & $4(5.71)$ & $45(64.29)$ \\
Imipenem & $68(97.14)$ & $1(1.43)$ & $1(1.43)$ \\
Meropenem & $65(92.86)$ & $1(1.43)$ & $4(4.29)$ \\
Nitrofurantoin & $39(55.71)$ & $4(5.71)$ & $27(38.57)$ \\
Ofloxacin & $26(37.14)$ & $0(0.00)$ & $44(62.86)$ \\
Piperacillin/tazobactam & $42(60.00)$ & $4(5.71)$ & $24(34.29)$ \\
Tetracycline & $0(0.00)$ & $0(0.00)$ & $70(100.00)$ \\
\hline
\end{tabular}

$S$ sensitivity, I intermediate, $R$ resistant

meropenem 65 (92.8\%), ertapenem 65 (92.86\%) and piperacillin/tazobactam $42(60.0 \%)$. None of the isolates were sensitive to ampicillin, chloramphenicol and tetracycline.

Sixty-six of the phenotypically confirmed ESBL producers contained at least one ESBL gene with no gene seen in 4 of the isolates. The $b l a_{\text {СТХ-M }}$ was the predominate gene seen (Fig. 1).

The most associated risk factors to infections caused by ESBL producing $E$. coli was previous antibiotics use in the past 3 months $(P=0.006)$, intensive care unit admission $(P=0.04)$, recent surgery $(P=0.015)$ (Table 4$)$.

\section{Discussion}

In this cross-sectional study, urine specimens accounted for the majority of $E$. coli positive specimens analysed. This fact is not surprising because urinary tract infections (UTI) have been established to be one of the commonest hospital-acquired infections in Nigeria and other parts of the world [9-11].

Our study revealed a high rate (70/200, 35.0\%) of ESBL producing E. coli among hospitalized patients in Enugu, three times the rate reported in an earlier study (11.9\%) in the same hospital in 2009 [12]. This increase within 8 years maybe due to unchecked antibiotics consumption especially beta-lactam antibiotics by the patients and poor infection prevention control measures. This finding is in agreement with what was reported in studies done in Bucharest (35.0\%) [13] and Zaria (34.3\%) [14]. Lower rates were reported in the South Eastern (16.0\%) [15] and
South Western (20.0\%) [16] parts of Nigeria. This shows that there are wide geographical variations in rates and even within the same hospital over time as seen in the study. The rate of ESBL producing E. coli was significantly higher in hospital acquired infections. Although majority of the samples were gotten from patients with hospital acquired infection, but many studies have also reported higher rates in hospital acquired infections compared to community acquired infections $[17,18]$. This maybe because of increase in the use of antibiotics and poor infection prevention and control measures.

The co-resistance seen in tetracycline, chloramphenicol and ampicillin has also been reported in other studies

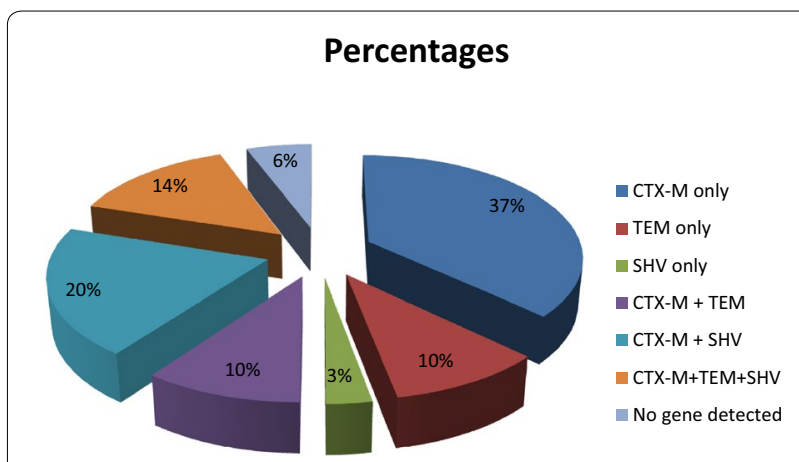

Fig. 1 Distribution of SHV, TEM and CTX-M genes among the positive isolates. ESBL genes detected in Escherichia coli isolates from different samples by multiplex polymerase chain reaction. The following genes were detected. Bla $a_{\text {CTXM }}$ only $37 \%, B l a_{\text {TEM }}$ only $10 \%, B l a_{\text {SHV }} 3 \%$, $B l a_{\text {CTX-M+SHV }} 20 \%, B l a_{\text {CTXM+TEM }} 10 \%, B / a_{\text {CTX }+ \text { TEM+SHV }} 14 \%$ 
Table 4 Multivariate analysis for risk factors associated with infections by ESBL producing Escherichia coli

\begin{tabular}{llll}
\hline Risk factor & Wald Chi-Square & OR & P value \\
\hline Female & 1.464 & 1.439 & 0.226 \\
Old age (60 years) & 1.324 & 0.681 & 0.250 \\
Recent surgery & 6.131 & 2.446 & 0.013 \\
Previous antibiotic ( $\leq 3$ months) & 7.461 & 1.948 & 0.006 \\
Beta-lactam & 9.850 & 1.555 & 0.002 \\
Aminoglycosides & 0.831 & 0.484 & 0.362 \\
Fluoroquinolones & 3.456 & 0.455 & 0.063 \\
Surgical ward & 0.064 & 0.976 & 0.806 \\
ICU ward & 3.502 & 1.432 & 0.041 \\
Medical ward & 0.511 & 0.788 & 0.475 \\
Orthopedic ward & 2.784 & 1.563 & 0.095 \\
Gynaecology ward & 0.933 & 0.872 & 0.334 \\
Post natal ward & 0.006 & 0.989 & 0.939 \\
Urinary Catheter & 1.661 & 2.002 & 0.197 \\
Nasogastric tubes & 1.961 & 0.789 & 0.161 \\
Central venous lines & 0.181 & 1.290 & 0.671 \\
Ventilator & 1.245 & 2.142 & 0.264 \\
Arterial lines & 0.323 & 0.538 & 0.570 \\
Endotracheal tube & 0.048 & 1.117 & 0.827 \\
Bronchoscopic tube & 1.478 & 0.574 & 0.224 \\
< 7 days stay in hospital & 2.228 & 0.487 & 0.136 \\
7-14days stay in hospital & 0.537 & 1.154 & 0.464 \\
> 14days stay in hospital & 3.065 & 1.412 & 0.080 \\
\hline OR odd ratio & &
\end{tabular}

OR odd ratio

[19-22]. These drugs are cheap and easily available so they are easily abused by the patients. The highest rate of resistance to available and easily affordable antimicrobial agents is of great concern. Nigeria does not have a strong regulated antibiotic prescription system, so antibiotics are easily purchased over the counter even from non-licensed dispensers, making them to be easily abused. Third generation cephalosporins, quinolones and aminoglycosides showed very poor activities. High rates of co-resistance to these antibiotics were reported in earlier studies [22-24]. Extended-spectrum beta-lactamase producing bacteria are known to carry genes conferring resistance to several non-beta lactam antibiotics and this would partly explain the high resistance rates to these drugs seen in this study. The resistance rates to third generation cephalosporins (ceftazidime $87.1 \%$, ceftriaxone $82.8 \%$ and cefotaxime $90.0 \%$ ) reported in this study were higher than the previous rates reported in the same hospital by Iroha et al. [12]. They reported $28.1 \%$ in ceftazidime, ceftriaxone $34.9 \%$ and cefotaxime $22.5 \%$. This highlights the increase in the spread of these resistance isolates in the hospital. Higher rates than what were obtained from this study have also been reported by Wani et al. [25] in India. There is superiority of local resistance data over regional, national or international data in the management of patients in any locality as it raises awareness to current resistance profile. Moderate resistances were seen in piperacillin/tazobactam and nitrofurantoin. Nitrofurantoin is a suitable, effective, and cheap alternative drug in the treatment of ESBL-producing $E$. coli related lower UTI [26]. Fortunately, carbapenems were found to be the most effective antibiotics against the ESBL producing $E$. coli, with imipenem being the most sensitive [27-29]. Carbapenems should be used with cautions because the overuse in the treatment of infections caused by ESBL producing organisms will gradually lead to acquisition and development of carbapenem resistant strains. There is a need to use alternatives such as nitrofurantoin and piperacillin/tazobactam as other options. This finding will help to guide development of effective antimicrobial stewardship and restriction policies.

Knowledge of the risk factors will help in early identification of patients likely to be infected with these organisms. This also is an important step in the prevention of the spread of resistant organisms among hospitalized patients, and visitors or relatives of the patients from their community. Control of the hospital spread will definitely have a role in minimising community spread of these resistant organisms. There is an association between previous exposures to antibiotics (especially beta-lactams) in the past 3 months to ESBL E. coli. This is consistent with previous studies showing that consumption of betalactams and fluoroquinolones are risk factors for predispositions to ESBL producing enterobacteriaceae carriage [30, 31]. Antibiotics misuse and abuse together with lack of knowledge of antibiotic resistance may be one of the major reasons for the increase in antibiotic resistance. There is a need to educate the prescribers and patients on rational and judicious use of antimicrobial agents. Other risk factors observed were admission in the intensive care unit and recent surgery. There are variations in the risk factors reported in different geographical locations. In Iran, Fatemeh et al. [9] reported recent surgery, prolonged antibiotic use, as the risk factors to infection caused by ESBL producing organisms.

This study confirmed that $b l a_{\mathrm{CTX}-\mathrm{M}}$ gene is the most common ESBL type harbored by E. coli strains in our hospital. This has been reported in other studies done in Nigeria [32-34]. This is because the plasmid carrying $b l a_{\text {СTX-M }}$ genes are known to carry other genes conferring resistance to several antibiotics. Secondly, the location of different resistance genes on single replicon leads to co-selection and may have contributed to the dissemination [35]. Yahaya et al. [27], in Maiduguri reported $b l a_{\mathrm{SHV}}$ (36.4\%) as the predominant gene followed by $b l a_{\mathrm{TEM}}$ (31.4\%) and bla ${ }_{\text {СТХ-M }}(27.3 \%)$. In Bangladesh, Yesmin 
et al. [36] reported $b l a_{\mathrm{TEM}}(50.5 \%)$ as the predominant gene followed by $b l a_{\mathrm{CTX}-\mathrm{M}}(46.7 \%)$ and $b l a_{\mathrm{SHV}}(18.7 \%)$. All these studies confirmed that gene predominance varied between regions and locations and to a large extent determine the resistance profiles of the organisms in the locality. It was also noticed in this study that some of the bacteria possessed multiple genes as had been established in some earlier studies $[15,37]$.

To the best of our knowledge, this is the first study that did a molecular study of ESBL genes in our institution. This knowledge will guide clinicians in developing antibiotics guidelines and policies, aimed at curbing the spread of ESBL E. coli. Further research on whole genomic sequencing is advocated to fully understand the epidemiology behind the spread of these organisms. We should be a step ahead in the management of multidrug resistant organisms in order to mitigate the evolution of the superbug that can defy all known antibiotics.

\section{Conclusion}

This study demonstrated a high prevalence $(35.0 \%)$ of extended spectrum beta lactamase producing $E$. coli in our hospital. There is a need for urgent interventions, including regular surveillance aimed at curbing the spread of extended spectrum beta lactamase producing E. coli, development of effective hospital antibiotics policies and effective infection prevention and control measures.

\begin{abstract}
Abbreviations
ATCC: American type culture collection; AOR: adjusted odds ratio; API: analytic profile index; CTX-M: cefotaximase-Munich; Cl: confidence interval; dNTP: deoxynucleoside triphosphates; ESBL: extended-spectrum beta-lactamase; PCR: polymerase chain reaction; SHV: sulfhydryl variable; SPSS: Statistical Package for Social Sciences; TEM: Temoniera; UNTH: University of Nigeria Teaching Hospital; UTI: urinary tract infection; WHO: World Health Organization.
\end{abstract}

\section{Acknowledgements}

Not applicable.

\section{Authors' contributions}

Conception and design of study: INN, MEO, SOE. Data collection: INN, SOE. Analysis and/or interpretation of data: INN, MEO, SOE, UCO. Drafting the manuscript: INN, MEO, UCO. Revising the manuscript critically for important intellectual content: INN, MEO, SOE, UCO. Approval of the version of the manuscript to be published: MEO, UCO. All authors read and approved the final manuscript.

\section{Funding}

This research received no specific grant from any funding agency in the public, commercial or not-for-profit sectors.

\section{Availability of data and materials}

The datasets used and/or analysed during this study are available from the corresponding author on reasonable request.

\section{Ethics approval and consent to participate}

The Health Research and Ethics committee of the University of Nigeria Teaching Hospital, Ituku-Ozalla, Enugu. Informed and written consent were obtained from the participate.
Consent for publication

Not applicable.

\section{Competing interests}

The authors declare that they have no competing interests.

Received: 16 October 2019 Accepted: 2 December 2019

Published online: 12 December 2019

References

1. Contou R, Gonzalez-Alba J, Galian J. CTX-M enzymes: origin and diffusion. Front Microbiol. 2012;2:110.

2. Livermore D. Current epidemiology and growing resistance of gram negative pathogens. Korean J Intern Med. 2012;27:128-42.

3. World Health Organization. Antimicrobial resistance. http://www.who.int/ gpsc/5feb/antimicrobial-resistance/en/. Accessed 25th Mar 2019.

4. Fang H, Ataker F, Hedin G, Dornbusch K. Molecular epidemiology of extended-spectrum-lactamases among Escherichia coli isolates collected in a Swedish hospital and its associated health care facilities from 2001 to 2006. J Clin Microbiol. 2008;46(2):707-12.

5. Endimiani A, Hujer A, Hujer K, Schriver A, Jacobs M, Rice L, et al. Evaluation of a commercial microarray system for detection of SHV, TEM, CTX-M and KPC-type beta lactamase genes in Gram negative isolates. J Clin Microbiol. 2010;48:2618-22.

6. Schwaber MJ, Carmeli Y. Mortality and delay in effective therapy associated with extended-spectrum beta-lactamase production in Enterobacteriaceae bacteraemia: a systematic review and meta-analysis. J Antimicrob Chemother. 2007;60:913-20.

7. Schwaber MJ, Navon-Venezia S, Kaye KS, Ben-Ami R, Schwartz D, Carmeli Y. Clinical and economic impact of bacteremia with extended-spectrum beta-lactamase-producing Enterobacteriaceae. Antimicrob Agents Chemother. 2006;50:1257-62.

8. Clinical and Laboratory Standards Institute. Performance standards for antimicrobial susceptibility testing; twenty-fifth informational Supplement (M100-S25). http://www.clsi.org/blog/2015/01/08/clsi-publishesnew-antimicrobial-susceptibility-testing-standards/. Accessed 15 Aug 2015.

9. Fatemeh A, Emran A, Elnaz K, Mohammad J, Mahboubeh N. The frequency of extended-spectrum beta-lactamase (ESBL) in Escherichia coli and Klebsiella pneumoniae: a report from Mashhad, Iran. J Med Bacteriol. 2013;2(1):12-9.

10. Oduro-Mensah D, Obeng-Nkrumah N, Bonney E, Oduro-Mensah E, Twum-Danso K, Osei Y, Sackey S. Genetic characterization of TEM-type ESBL-associated antibacterial resistance in enterobacteriaceae in a tertiary hospital in Ghana. Ann Clin Microbiol Antimicrob. 2016;15:29.

11. Horsefall S, Abbey S, Okonkwo I. Prevalence of extended-spectrum beta-lactamase and plasmid status of Escherichia coli and Klebsiella pneumoniae from clinical sources in UPTH, Port Harcourt, Nigeria. NY Sci J. 2017;10(3):29-39.

12. Iroha I, Adikwu M, Esimone C, Aibinu I, Amadi E. Extended spectrum betalactamase (ESBL) in E. coli isolated from a tertiary hospital in Enugu state, Nigeria. Pak J Sci. 2009;25:279-88.

13. Hristea A, Olaru ID, Adams-Sapper S, Riley LW. Characterization of ESBLproducing Escherichia coli and Klebsiella pneumoniae from bloodstream infections in three hospitals in Bucharest, Romania: a preliminary study. Infect Dis. 2015;47(1):46-51.

14. Giwa FJ, Ige OT, Haruna DM, Yaqub Y, Lamido TZ, Usman SY. ExtendedSpectrum beta-lactamase production and antimicrobial susceptibility pattern of uropathogens in a Tertiary Hospital in Northwestern Nigeria. Ann Trop Pathol. 2018;9:11-6.

15. Akujobi C, Ewuru C. Detection of extended spectrum $\beta$-lactamases in gram negative bacilli from clinical specimens in a teaching hospital in south eastern Nigeria. Niger Med J. 2010;51:141-6.

16. Aibinu I, Ohaegbulam V, Adenipekun IE, Ogunsola F, Odugbemi T, Mee B. Extended-spectrum beta-lactamase enzymes in clinical isolates of Enterobacter species from Lagos Nigeria. J Clin Microbiol. 2003:41(5):2197-200.

17. Latifpour M, Gholipour A, Damavandi MS. Prevalence of extendedspectrum beta-lactamase-producing Klebsiella pneumoniae isolates in 
nosocomial and community-acquired urinary tract infections. Jundishapur J Microbiol. 2016;9:e31179.

18. Khanfar HS, Bindayna KM, Senok AC, Botta GA. Extended spectrum betalactamases (ESBL) in Escherichia coli and Klebsiella pneumoniae: trends in the hospital and community settings. J Infect Dev Ctries. 2009;3:295-9.

19. Iregbu K, Anwaal U. Extended spectrum beta-lactamases producing Klebsiella pneumoniae septicaemia outbreak in the in the neonatal intensive unit of a tertiary hospital in Nigeria. Afr Med Sci. 2007;36:225-88.

20. Peirano G, Hun King Sang J, Pitondo-Silva A, Laupland K, Pitout J. Molecular epidemiology of extended-spectrum- $\beta$-lactamase-producing Klebsiella pneumoniae over a 10 years period in Calgary, Canada. J Antimicrob Chemother. 2012;67:1114-20.

21. Abbott S. Klebsiella, Enterobacter, Citrobacter and Serratia. In: Murray PR, editor. Manual of clinical microbiology. 9th ed. Washington DC: ASM Press; 2007

22. Oli AN, Eze DE, Gugu TH, Ezeobi I, Maduagwu UN, Ihekwereme CP. Multi-antibiotic resistant extended-spectrum beta-lactamase producing bacteria pose a challenge to the effective treatment of wound and skin infections. Pan Afr Med J. 2017;27:66.

23. Akanbi B, Ojonuba B, Njoku R. Detection of extended-spectrum beta lactamase producing Klebsiella pneumoniae and Escherichia coli in two hospitals in the federal capital territory Abuja, Nigeria. Open J Med Microbiol. 2013:3(4):207-12.

24. Kesavaram P, Krishnan P, Sikhamani R, Multidrug resistant CTX-M-producing Escherichia coli: a growing threat among HIV patients in India. J Pathog. 2016; 2016: 6 pages.

25. Wani K, Thankur M, Fayaz A, Fomdia B, Gulnaz B, Maroof P. Extended spectrum beta lactamase mediated resistance in $E$. coli in a tertiary hospital. Int J Health Sci. 2009:3:155-63.

26. Tasbakan MI, Pullukcu H, Sipahi OR, Yamazhan T, Ulusoy S. Nitrofurantoin in the treatment of extended-spectrum lactamase-producing Escherichia coli-related lower urinary tract infection. Int J Antimicrob Agents. 2012:40:554-6.

27. Yahaya M, Gadzama G, Zailani S, Aboderin A. Characterization of extended-spectrum beta-lactamase from Escherichia coli and Klebsiella species. J Clin Diagn Res. 2016;10(2):7-10.

28. Meeta S, Sati P, Preeti S. Prevalence and antibiogram of extendedspectrum beta-lactamase producing Gram negative bacilli and further molecular characterization of ESBL producing Escherichia coli and Klebsiella spp. J Clin Res. 2013;7(10):2168-72.
29. Adeyankinnu F, Babatunde O, Akinduti A, Aboderin B, Ogiogwa J, Agunlejika R. A multicenter study of beta lactamase resistant Escherichia coli and Klebsiella pneumoniae reveals high level chromosome mediated extended spectrum beta lactamase resistance in Ogun state, Nigeria. Interdiscip Perspect Infect Dis. 2014. https://doi.org/10.1155/2014/81989 6.

30. Augustine MR, Testerman TL, Justo JA, et al. Clinical risk score for prediction of extended spectrum beta-lactamase producing enterobacteriaceae in blood stream isolates. Infect Control Hosp Epidemiol. 2017:38:266-72.

31. Tumbarello M, Trecarichi EM, Bassetti M, et al. Identifying patients harbouring ESBL producing enterobacteriaceae on hospital admission: derivation and validation of a scoring system. Antimicrob Agents Chemother. 2011:55:3485-90

32. Raji M, Ojemeh O, Rotimi O. Sequence analysis of genes mediating extended-spectrum beta-lactamase production in isolates of enterobacteriaceae in Lagos Teaching Hospital. BioMed Cent Infect Dis. 2015;15:259.

33. Ogefere $H$, Aigbiremwen $P$, Omoregie R. Extended-spectrum betalactamase producing Gram negative isolates from urine and wound specimens in a tertiary health facility in Southern Nigeria. Trop J Pharma Res. 2015;14(6):1089-92.

34. Rani S, Jahnavi I, Nagamani K. Phenotypic and molecular characterization of ESBLs producing enterobacteriaceae in A Tertiary care hospital. J Dent Med Sci. 2016;15(9):27-34.

35. Canton R, Coque TM. The CTX-M $\beta$-lactamase pandemic. Curr Opin Microbiol. 2006;9:466-75.

36. Yesmin T, Hossain A, Paul S, Yusuf A, Sultana S, Gmowla G. Detection of extended-spectrum beta-lactamases producing genes among third generation cephalosporins sensitive bacteria strains from a medical college hospital in Bangladesh. J Allergy Disord Ther. 2014;1:1.

37. Bali E, Leyla A, Nedim S. Phenotypic and molecular characterization of SHV, TEM, CTX-M and extended-spectrum $\beta$-lactamase produced by Escherichia coli, Acinetobacter baumannii and Klebsiella isolates in a Turkish hospital. Afr J Microbiol Res. 2010;4(8):650-4.

\section{Publisher's Note}

Springer Nature remains neutral with regard to jurisdictional claims in published maps and institutional affiliations.
Ready to submit your research? Choose BMC and benefit from:

- fast, convenient online submission

- thorough peer review by experienced researchers in your field

- rapid publication on acceptance

- support for research data, including large and complex data types

- gold Open Access which fosters wider collaboration and increased citations

- maximum visibility for your research: over 100M website views per year

At BMC, research is always in progress.

Learn more biomedcentral.com/submissions 